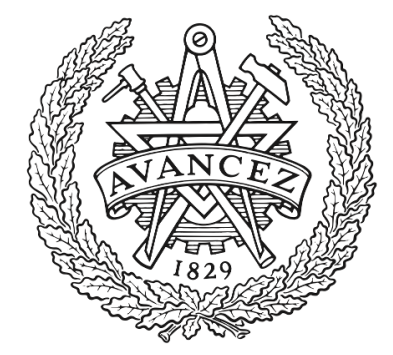

\title{
CHALMERS
}

UNIVERSITY OF TECHNOLOGY

\section{Hot-Cavity Spectroscopy of Dark Pulse Kerr Combs in Microresonators}

Downloaded from: https://research.chalmers.se, 2023-04-26 01:11 UTC

Citation for the original published paper (version of record):

Nazemosadat Arsanjani, S., Fülöp, A., Helgason, Ò. et al (2019). Hot-Cavity Spectroscopy of Dark Pulse Kerr Combs in Microresonators. 2019 Conference on Lasers and Electro-Optics Europe and European Quantum Electronics Conference, CLEO/Europe-EQEC 2019, June 2019.

http://dx.doi.org/10.1109/CLEOE-EQEC.2019.8873415

N.B. When citing this work, cite the original published paper. 


\title{
Hot-Cavity Spectroscopy of Dark Pulse Kerr Combs in Microresonators
}

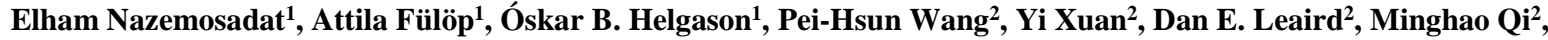 \\ Enrique Silvestre $^{3}$, Andrew M. Weiner ${ }^{2}$, and Victor Torres-Company ${ }^{1}$
}

1. Photonics Laboratory, Department of Microtechnology and Nanoscience (MC2), Chalmers University of Technology, Göteborg, Sweden 2. School of Electrical and Computer Engineering, Purdue University, West Lafayette, IN 47907-2035, USA

3. Department of Optics-ICMUV, University of Valencia, 46100 Burjassot, Valencia, Spain

Kerr frequency combs are generated through cascaded four-wave mixing in high-Q microresonators [1]. These devices are pumped with a continuous-wave laser and modulational instability (MI) is responsible for the growth of the initial comb lines. Since it is easier to satisfy the MI phase matching condition in the anomalous dispersion regime, most studies on Kerr combs have focused on anomalous dispersion microresonators. However, coherent microresonator combs can also take place in the normal dispersion regime. In these combs, phase matching is attained with the aid of the mode coupling between transverse modes of the microresonator [2]. One particularly interesting comb state that operates in the normal dispersion regime is the dark pulse Kerr comb [3]. The time domain pulses of these combs arise as interlocking switching waves that connect the upper and lower homogenous steady state solutions of the bi-stability curve in the continuous-wave-driven Kerr cavity [see Fig. (a)] [3]. These combs are of high interest as most nonlinear materials suitable for fabricating microresonators display normal dispersion in the visible and near infrared ranges. Moreover, these combs provide a much higher power conversion efficiency compared to bright-soliton combs, which makes them particularly useful for telecommunications [4]. Nevertheless, the formation of dark-pulse combs and their dynamics remain largely unexplored. In this work, we provide a better physical understanding of the formation of this type of combs by measuring the system's response as the pump laser is tuned into resonance. We discover that the formation of the dark pulse Kerr combs is associated with the appearance of an extra resonance, in striking similarity to recent observations made for bright temporal solitons in the anomalous dispersion regime [5], and explain the findings based on cross-phase modulation (XPM) induced by the two homogeneous steady state solutions of the Kerr cavity.

We use the same high-Q silicon nitride microresonator as in [4]. The pump laser has an on-chip power of $\sim 100 \mathrm{~mW}$ and is tuned over the cold-cavity resonances of both linearly coupled transverse modes from the thermally stable blue side towards the red. We measure the system's response with the aid of a red-detuned probe laser that is fixed in frequency. This laser is weakly modulated with an external electro-optic intensity modulator driven by an external radio-frequency source. The sidebands are swept with the RF source and the response measured by a vector network analyzer for different detunings of the pump laser [Fig. (b)]. Only one of the sidebands is swept across the cavity resonances. Figure (c) displays the results. As the pump is tuned into resonance, the resonances of the two linearly coupled modes are shifted to longer wavelengths due to thermal effects. Interestingly, once the dark pulse comb is generated, a new resonance emerges [Fig. (c)]. The modelocked state breaks the time invariance response of the system. Since the Kerr phase shift is intensity dependent, the high and low intensity levels of the dark pulse induce different phase shifts on the cavity resonance, leading to two separate resonances. This double-resonance is a unique property of soliton states in Kerr microresonators [5] which had not been demonstrated in dark-pulse combs to date.
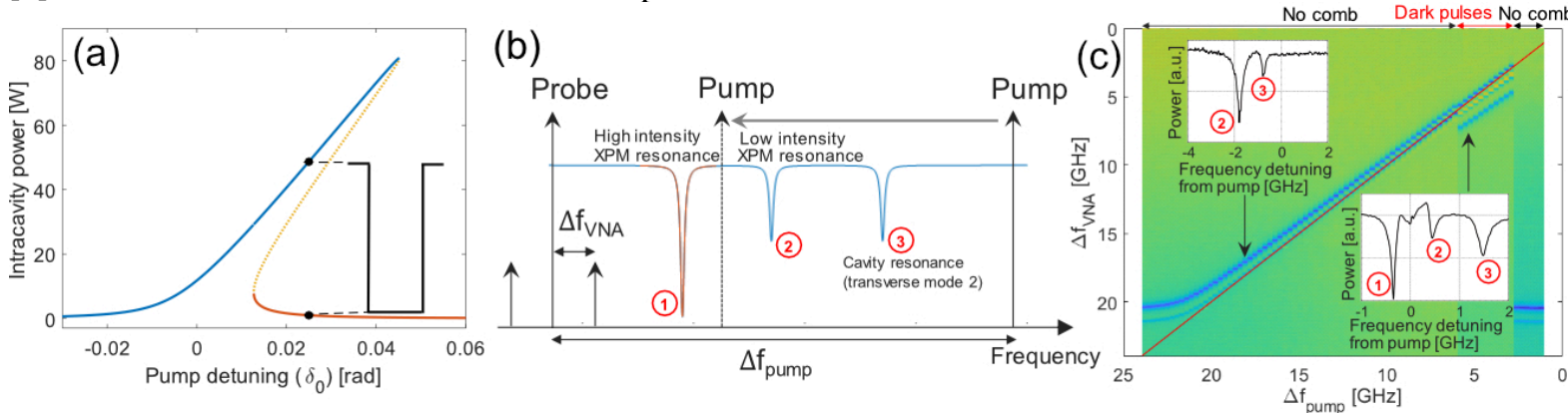

Fig. (a) Bi-stability curve as a function of the pump detuning. (b) Scheme used for measuring the system's response, showing the cavity resonances of the two linearly coupled transverse modes and a newly generated resonance that is only formed in the dark pulse comb regime. (c) The measured VNA response of the cavity for different pump detunings. Insets show the VNA response for specific detunings indicated by arrows; the formation of a new

\section{References} resonance in the dark pulse regime can be clearly seen.

[1] Kippenberg, et al., "Microresonator-based optical frequency combs,” Science 332, 555 (2011).

[2] Y. Liu, et al., "Investigation of mode coupling in normal-dispersion silicon nitride microresonators for Kerr ...,” Optica 1, 137 (2014).

[3] X. Xue, et al., "Mode-locked dark pulse Kerr combs in normal-dispersion microresonator,” Nature Photon. 9, 594-600 (2015).

[4] A. Fülöp, et al., "High-order coherent communications using mode-locked dark-pulse Kerr ...,” Nature Commun. 9, 1598 (2018).

[5] H. Guo, et al. "Universal dynamics and deterministic switching of dissipative Kerr solitons ...,” Nat. Physics 13, 94-102 (2017). 